ACCEPTED MANUSCRIPT

\title{
Photoacoustic imaging in percutaneous radiofrequency ablation: device guidance and ablation visualization
}

To cite this article before publication: Francis Kalloor Joseph et al 2019 Phys. Med. Biol. in press https://doi.org/10.1088/1361-6560/ab36a1

\section{Manuscript version: Accepted Manuscript}

Accepted Manuscript is "the version of the article accepted for publication including all changes made as a result of the peer review process, and which may also include the addition to the article by IOP Publishing of a header, an article ID, a cover sheet and/or an 'Accepted

Manuscript' watermark, but excluding any other editing, typesetting or other changes made by IOP Publishing and/or its licensors"

This Accepted Manuscript is $\odot 2019$ Institute of Physics and Engineering in Medicine.

During the embargo period (the 12 month period from the publication of the Version of Record of this article), the Accepted Manuscript is fully protected by copyright and cannot be reused or reposted elsewhere.

As the Version of Record of this article is going to be / has been published on a subscription basis, this Accepted Manuscript is available for reuse under a CC BY-NC-ND 3.0 licence after the 12 month embargo period.

After the embargo period, everyone is permitted to use copy and redistribute this article for non-commercial purposes only, provided that they adhere to all the terms of the licence https://creativecommons.org/licences/by-nc-nd/3.0

Although reasonable endeavours have been taken to obtain all necessary permissions from third parties to include their copyrighted content within this article, their full citation and copyright line may not be present in this Accepted Manuscript version. Before using any content from this article, please refer to the Version of Record on IOPscience once published for full citation and copyright details, as permissions will likely be required. All third party content is fully copyright protected, unless specifically stated otherwise in the figure caption in the Version of Record.

View the article online for updates and enhancements. 


\title{
Photoacoustic Imaging in Percutaneous
} Radiofrequency Ablation: Device Guidance and Ablation Visualization

\author{
Kalloor Joseph Francis \& Srirang Manohar
}

Biomedical Photonic Imaging Group and Multimodality Medical Imaging Group, Faculty of Science and Technology, Technical Medical Center, University of Twente, PO Box 217, 7500 AE Enschede, The Netherlands

E-mail: s.manohar@utwente.nl

July 2019

\begin{abstract}
Percutaneous radiofrequency ablation (RFA) is gaining importance as a locoregional treatment for tumors in several organs including the liver, lung, kidney and bone. In RFA, the tumor is eradicated with the direct application of heat using alternating current through a needle electrode positioned under imaging guidance. Various imaging methods are used in the RFA ablation procedure but these have drawbacks. In this work, we introduce photoacoustic (PA) imaging as a new method with potential to visualize the targeting of RFA needle into a region of interest and to report on the extent of ablation achieved. We demonstrate the proof-of-concept in using PA imaging together with ultrasound imaging on ex vivo biological samples in the laboratory simulating relevant clinical scenarios in RFA. These include guidance of the RFA needle to target tissue, mapping of simulated blood vessels during needle insertion and differentiation between ablated and surrounding tissue. The results of this first investigation into the use of PA imaging to assist RFA procedures are encouraging. We discuss the challenges encountered, the scope for future work and envisaged clinical application.
\end{abstract}

Keywords: Radiofrequency Ablation, Photoacoustic, Ultrasound, Surgical tool tracking, Liver treatment, ex vivo, Real-time Imaging

\section{Introduction}

With advances in surgical devices and in medical imaging technologies, minimally invasive treatment methods are gaining ground and are being offered as alternatives to open surgery in thoracic and abdominal oncology [1]. Several locoregional treatment techniques such as thermal ablation using microwave, radiofrequency, cryo, focused ultrasound, and laser energies; chemical ablation using acetic acid or ethanol; radiation using brachytherapy are increasingly being accepted as treatment methods 


\section{Photoacoustic Imaging in Percutaneous Radiofrequency Ablation}

for malignancies in the liver, breast, kidney, lungs, prostate and soft tissues [2]. The benefits of short hospitalization, high post-treatment health-related quality of life, and greater survival rate make Radiofrequency ablation (RFA) the treatment of choice for small (less than $3 \mathrm{~cm}$ ) and solitary tumors [3]. In RFA, a needle electrode is placed inside the tumor volume under image guidance. A grounding pad is attached on the surface of the tissue to complete the electrical circuit. An alternating current between $350-500 \mathrm{kHz}$ applied to the electrodes induces ionic agitation in the tissue around the needle electrode. This results in frictional heating of the tissue; when administered with appropriate temperature and treatment duration this can cause coagulative necrosis of targeted tissue [4]. RFA is widely used for cancer treatment in lung, liver, kidney, breast and bone $[5,6]$.

The main limitation of RFA is its high tumor recurrence rate [7] which is largely due to incomplete tumor ablation. This is due to inadequate achievement of cytotoxic temperature over the entire tumor volume. The reasons for theincomplete ablation may be found in the intrinsic tissue heating mechanism of the RFA technology which is a twostep process: tissue in the close proximity of the applicator undergoes resistive heating, followed by conduction heat transfer to the surrounding. While the rise in temperature in the tissue undergoing resistive heating is fast, the surrounding tissue exposed to conductive heat transfer experiences a slower temperature rise. To achieve the required temperature over the spatial extent of the tumor, the electrode-tissue interface can result in temperatures above $100^{\circ} \mathrm{C}$ resulting in desiccation, causing an abrupt increase in impedance [2], and lower electric current flow into tissue. Other factors that can lead to incomplete tumor ablation are inadequacies in image guidance and ablationassessment [8]. Much work is being done to address the intrinsic limitations of RFA such as with the use of improved applicators, and protocols to avoid desiccation [2]. We limit our discussion to factors to improve in image- guidance during RFA procedures, which may be also beneficial for other minimally-invasive ablation modalities.

Imaging modalities are vital in percutaneous RFA procedures for identifying target tissue, accurate probe placement and in monitoring the treatment [9, 10]. Several imaging modalities are being used to assist RFA procedures such as Computed Tomography (CT), Magnetic Resonance Imaging (MRI) and Ultrasound (US) imaging [10]. The selection of imaging modality depends on factors like the visibility, number and the size of the tumors, surrounding tissues and organs, the availability of imaging system and also the convenience to perform the procedure. CT is used for pre- and post-ablation imaging to plan the procedure and to determine the success of the treatment [10]. Contrast-enhanced CT can clearly discriminate the ablation boundary due to increased vascularity around the thermal lesion [10]. However, factors like non-availability of realtime CT system for device guidance, the need for contrast agents to visualize ablation, the large size of the instrument, limited access to the targeted treatment volume and the risk of ionizing radiation limits its use during the procedure [10, 11]. MRI is considered the most accurate modality for RFA application [10]. MRI can be used to image the RFA device along with landmarks in the target organ, the functional capability to map water 


\section{Photoacoustic Imaging in Percutaneous Radiofrequency Ablation}

molecule distribution helps to differentiate tumor (hyperintense), ablated (hypointense) and normal tissue [10]. The need for an open MRI system, MRI compatible materials for surgical tools, and contrast agents limit its real-time use in monitoring the RFA procedure. Although MRI and CT have clear advantages in guiding RFA procedures, the key benefit of RFA as a bedside, outpatient treatment procedure is lost with these modalities. US imaging solves the above-mentioned problems being low-cost, portable, readily available and real-time [12]. Hence, percutaneous or laparoscopic US-guided RFA procedures are widely used in clinics. Several associated technologies like Doppler US for blood vessel mapping and the contrast-enhanced US for ablated tissue visualization are also in use with RFA [13].

For ultrasound image-guided RFA procedures, tumor recurrence is associated with improper needle placement and incomplete ablation which are linked to the size and the location of the tumor [14]. This insight has been gained in the study by Mulier et al where local recurrence for laparoscopic and percutaneous US-guided RFA procedures for two range of tumor sizes, small $(<3 \mathrm{~cm})$ and large $(3-5 \mathrm{~cm})$ are compared [7]. Local recurrence under laparoscopic US-guided RFA ranges from 3.6\% (small) to $21.7 \%$ (large), while for percutaneous procedure it is from $16 \%$ (small) to $25.9 \%$ (large) [7]. The deeper the tumor lies with respect to the transducer, the higher the chances of improper needle placement [7]. Further, the larger the tumor, the harder it is to estimate the amount of ablation under US imaging [8]. An additional factor which contributes to incomplete ablation is the presence of blood vessels near the thermal lesion, resulting in heat losses to the circulating blood, which are not accounted for when the vessels are not detected with US [8].

A method which can address some of the above issues as an add-on to US imaging is photoacoustic (PA) imaging. This is a relatively new technology which combines optical absorption contrast and ultrasound resolution. In PA imaging, short laser pulses generate thermoelastic expansion at optical absorber sites resulting in a pressure rise leading to acoustic waves which can be detected for imaging $[15,16]$. PA imaging can potentially serve as an adjunct to US imaging in guiding RFA procedures because of the following reasons. (i) Optical absorption of metals are high compared to tissue allowing visualization of needles [17]. (ii) Optical absorption of oxyhemoglobin and deoxyhemoglobin is high compared to tissue allowing visualization of blood vessels [18]. (iii) An optical absorption and scattering contrast is present between tumor and healthy tissue [19]. (iv) Optical property changes occur during and after ablation [20, 21, 22, 23].

In order to use PA imaging for RFA, a combination of the above aspects needs to be incorporated in a percutaneous RFA setting. In this context, we encounter the requirement of visualizing $\mathrm{RFA}$ needle, localizing the target tumor, identifying large blood vessels which need to be avoided while planning the trajectory of the needle, visualizing the deployment of the multiple electrode tines and reporting on the extent of the ablated zone. While visualization of metal needles using PA imaging has been reported previously for drug delivery, biopsy and brachytherapy [24, 17, 25], imaging a multi-electrode RFA device has not been reported, which is challenging considering the 


\section{Photoacoustic Imaging in Percutaneous Radiofrequency Ablation}

small size of the electrode tines. Further, while a few previously several groups have studied PA imaging of ablated cardiac superficial tissue [20, 21, 22] and observed a high PA intensity this has not been well studied for other tissue types like liver and kidney, where image guidance needs to be improved $[26,23]$. In this work, we have attempted to address the above aspects.

We report on the first proof-of-concept investigation into the use of PA imaging in combination with US for the RFA procedure. This is performed in the laboratory where we simulate the clinical setting on ex vivo tissues. We study three specific clinically relevant scenarios for PA imaging: first, in the guidance of the RFA needle to an anomalous target tissue, second, in the visualization of blood vessels during the guiding procedure, and third in the visualization of the ablated regions.

\section{Methods}

The RFA procedures involve guiding the needle electrode into the target tissue and ablating the tissue. To test the feasibility of using PA imaging for this RFA procedure, we conducted experiments to answer the following questions.

(i) Can PA imaging improve the visibility of RFA needle in a percutaneous setting in comparison with standard US imaging? If so, how is the needle visualization with PA imaging differ from US at different angles of insertion? Is it possible to visualize the needles tines, which are known to be difficult to visualize in US imaging?

(ii) Is it possible to use real-time PA imaging to guide the RFA needle to an anomalous tissue which differs in its optical properties compared to normal tissue?

(iii) Can blood vessels be mapped during RFA needle guidance using PA imaging?

(iv) How well can we use PA imaging in differentiating ablated and native tissue? Experiments are designed to address the above questions. In this section the details of imaging and ablation setup used in the experiments are provided.

\subsection{Photoacoustic and Ultrasound Imaging System}

A commercial US system, Alpinion Ecube 12R (Alpinion Medical Systems, South Korea) was used for the real-time US and PA imaging. The L3 - 12 transducer with center frequency $8.5 \mathrm{MHz}$ was used as the ultrasound detector. For PA excitation, we used an Nd:YAG Q-switched laser (Quanta Ray Lab 170, Spectra-Physics, USA) pumping an OPO (versaScan L-532, GWU, Germany). The laser beam was coupled to a fiber bundle which splits into 7 bundles of $4 \mathrm{~mm}$ diameter each, at the distal end. A 3D printed holder was used to carry the linear ultrasound transducer with three fiber bundles on either side, along the long axis of the transducer (Fig. 1); the 7th bundle is used for monitoring the laser pulse energy. The angle and distance between the transducer and fiber bundles were chosen to geometrically have (i) the beams intersecting at the focal point of the transducer $(20 \mathrm{~mm}$ ), and (ii) make a minimal angle between the beams and the imaging plane. 


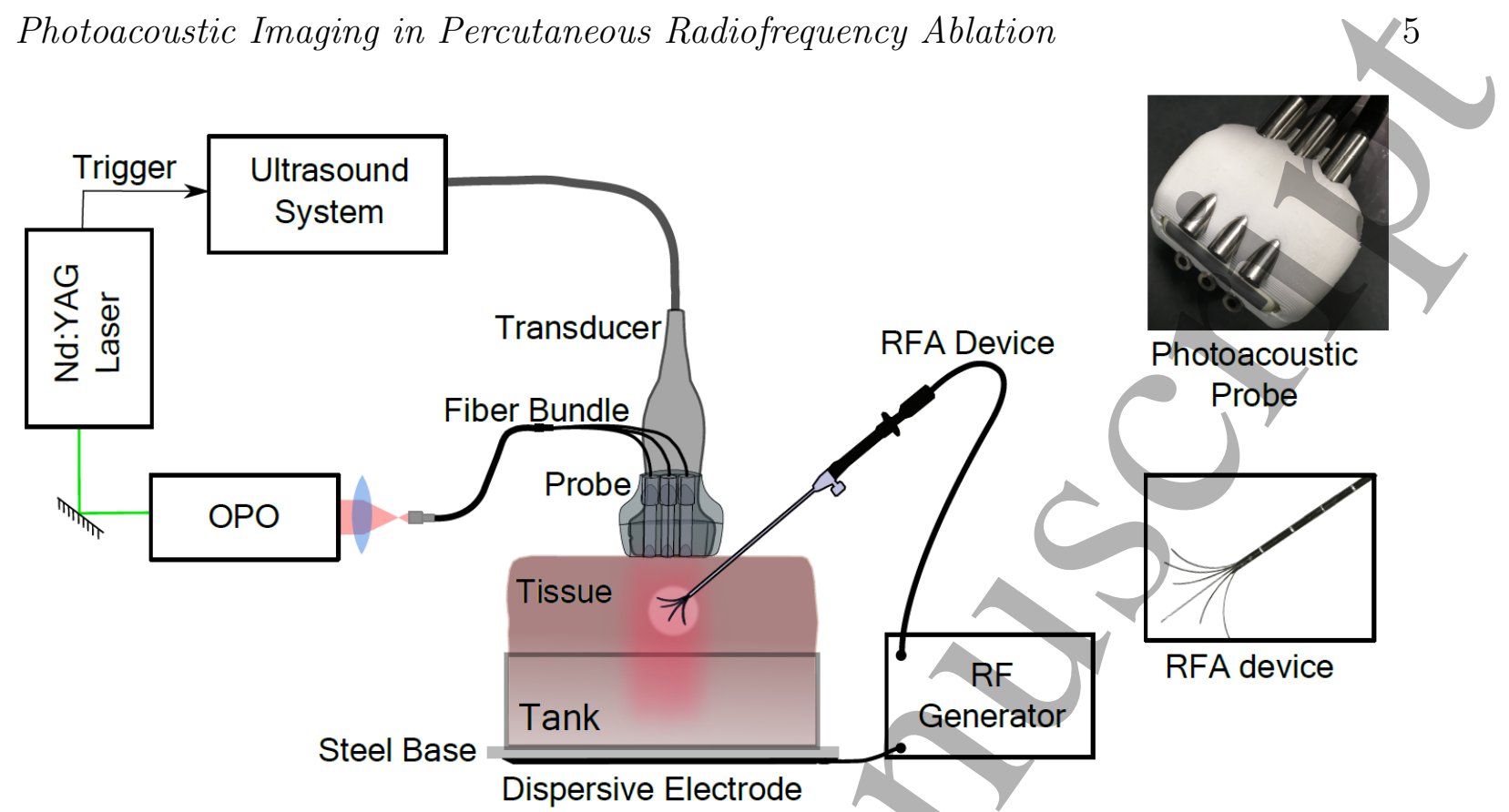

Figure 1. Photoacoustic imaging system configuration for ex vivo studies of radiofrequency ablation (RFA) procedure. A probe holding the fiber bundle and the transducer was used for imaging.

A custom acquisition sequence was used to perform the combined US and PA imaging. The sequence starts with an external synchronization pulse from the laser triggering the PA signal acquisition followed by a $20 \mathrm{~ms}$ waiting time and a focused B-Mode US imaging. A built-in real-time reconstruction from the ultrasound system was used for image formation. The reconstruction is performed in three stages. First, received RF signals are apodized and beamformed by applying appropriate delays and summation. In the second stage demodulation filtering is applied, where DC component in the beamformed data is removed, time gain compensation is included, followed by quadrature demodulation, lowpass filtering and subsampling. Finally, the output of the demodulation stage is envelope detected, log compressed and scaled to the dynamic range before displaying [27].

\subsection{Radiofrequency Ablation System}

A clinically valid RFA system (Angiodynamics, USA) was used in this laboratory study. The RF generator (1500X) operating at $460 \mathrm{KHz}$ drives a unipolar electrode (StarBurst XL) with an adjustable 9 element (tine) array. A custom developed water tank with steel base was used to hold the tissue, with the dispersive electrode from the RF generator attached to the metal base (Fig. 1). An in-house developed needle holder with protractor was used to insert the RFA needle in a sample with a specific angle and to a specific depth. 


\section{Photoacoustic Imaging in Percutaneous Radiofrequency Ablation}

\subsection{RFA Needle Guidance}

Three experiments were conducted to study the capability of PA imaging to visualize and guide the RFA needle into chicken breast tissue.

In the first experiment, we studied RFA needle visualization at different angles with respect to the transducer. Three slices of chicken breast tissue with an approximate thickness of $15 \mathrm{~mm}$ each were stacked in the tank to form the imaging volume. US gel was smeared on the tissue surface for acoustic coupling and the PA probe was mounted on top of the sample (Fig. 1). For all the RFA needle visualization experiments, a wavelength of $720 \mathrm{~nm}$ was used with measured energy of $9.2 \mathrm{~mJ}$ for the six illuminating fiber bundles. This measured pulse energy provides an exposure of $11.8 \mathrm{~mJ} / \mathrm{cm}^{2}$ which is less than the permissible limit of $20 \mathrm{~mJ} / \mathrm{cm}^{2}$ [28]. The RFA needle was inserted at three different angles, $0^{0}, 15^{0}$ and $30^{0}$ through the tissue to the focus of the transducer $(20 \mathrm{~mm})$. The RFA needle has a trocar of $2.1 \mathrm{~mm}$ in diameter, and multiple array electrodes or tines each $0.4 \mathrm{~mm}$ in diameter and variable in length to a maximum of 50 mm when fully deployed. Due to the difference in surface area, PA signals were weaker from the tines compared to the trocar. To improve the absorption of the tines, black ink (permanent marker Pentel N50) was used to coat the surface of tines. The tines were deployed to approximately $15 \mathrm{~mm}$ in diameter. PA and US imaging were conducted and the beamformed images from Alpinion system were acquired.

In the second experiment, needle guidance to an absorbing target tissue mimicking a tumor was performed. The insert tissue was developed using a chicken tissue of 15 $\mathrm{mm}$ in diameter, dipped in India ink and air-dried for 30 mins. The tissue with ink was inserted between slices of chicken tissue and positioned around the focal point of the transducer. Photoacoustic assisted needle guidance to the target insert was performed and the tines were deployed to the target volume. Real-time PA and US images were acquired during the needle guidance.

In the third experiment, to mimic RFA needle guidance near blood vessels a nylon tube filled with human blood was used. The blood sample was extracted from a healthy volunteer and preserved in vacuette tube with heparin as anticoagulant. Total hemoglobin mass was $14.1 \mathrm{~g} / \mathrm{dL}$ and the oxygen saturation was measured using an avoximeter (ITC, USA). To understand the change in PA signal at a different oxygen saturation, the blood was exposed to air to achieve $40 \%$ gain in oxygen concentration. PA images were acquired at $760 \mathrm{~nm}$ with RFA needle in place and oxygen saturation of $47 \%$ and $88 \%$ in the tube.

\subsection{Application in Liver and Ablation Lesion Visualization}

The RFA procedure in the liver involves percutaneous needle guidance and ablation of the malignant tissue. Two experiments were conducted to (i) study PA assisted needle guidance into the liver and (ii) explore the differences in PA signal from ablated and native liver tissue.

In the first experiment, an ex vivo tissue sample was prepared with a layer of chicken 


\section{Photoacoustic Imaging in Percutaneous Radiofrequency Ablation}

breast tissue stacked atop the bovine liver. The chicken tissue was used to mimic the soft tissue above the liver in percutaneous procedures. The coating of the tines, and parameters of the laser excitation were the same as in previous experiments. Real-time PA and US images were acquired while guiding the needle to the liver.

To understand the nature of PA signals from ablated and native tissue, a preliminary study was conducted with an ex vivo bovine liver sample. The liver/sample was ablated based on standard procedure for the device, at a target temperature of $105^{0} \mathrm{C}$, for a duration of 5 minutes to form a $15 \mathrm{~mm}$ diameter lesion [29]. A slice (approximately $5 \mathrm{~mm}$ thick) of the ablated sample was extracted and mounted in a water tank using a polyethylene $\left(\operatorname{Saran}^{\mathrm{TM}}\right)$ film. The tank was then filled with a Phosphatebuffered saline (1X PBS) and Intralipid solution. PBS was used to preserve the nature of the tissue while the Intralipid solution was used to scatter the laser light and to get a uniform illumination at the focal point of the transducer. Additionally, the solution acted as a coupling medium for the US waves. The laser energy of $8.6 \mathrm{~mJ}$ (exposure of $11 \mathrm{~mJ} / \mathrm{cm}^{2}$ ) at $760 \mathrm{~nm}$ wavelength was used to image the sample. The wavelength was chosen to target the combined absorption from both oxy and deoxy-hemoglobin in the sample. Three dimensional PA and US images were acquired in a C-scan by sequentially positioning the linear array in steps of $1 \mathrm{~mm}$ along its short axis to cover an area of 25 mm.

\section{Results}

The results of our experiments are presented in this section and they are arranged in the same order as the research questions posed in Section 2.

\subsection{RFA Needle Visualization at Different Angles}

The first experiment was conducted to study how PA imaging can enhance RFA needle visualization at different angles. Figure 2 shows the results from US and PA imaging of the RFA needle inserted at $0^{\circ}, 15^{\circ}$ and $30^{\circ}$ with the tines deployed to $15 \mathrm{~mm}$ diameter. The schematic in Fig. 2 (a - c) shows the position and angle of the RFA needle inside the tissue. Figure $2(\mathrm{~d}-\mathrm{f})$ shows ultrasound images at $0^{0}, 15^{0}$ and $30^{0}$ respectively. It can be observed that the visibility of the RFA needle under US imaging drops significantly with increasing angle of insertion. Additionally, the visibility of the tines is poor in US images. Figure 2 ( $\mathrm{g}-\mathrm{i}$ ) shows the PA images of the RFA needle at different angles. For all the angles used in the experiment, high PA intensity is observed from the RFA needle. The number of tines visible under PA images were six at $0^{0}$, five at $15^{0}$ and four at $30^{\circ}$ inclination of the needle. The number of tines and the extent of its visibility in PA images are high compared to US images. The Contrast to Noise Ratio (CNR) of both PA and US images with the RFA trocar and tines are presented in Table. 1. A gain in contrast of 5.3 to 7.5 was observed for the tines in the PA images compared to the US images. While the contrast difference between PA and US images for trocar 
Photoacoustic Imaging in Percutaneous Radiofrequency Ablation

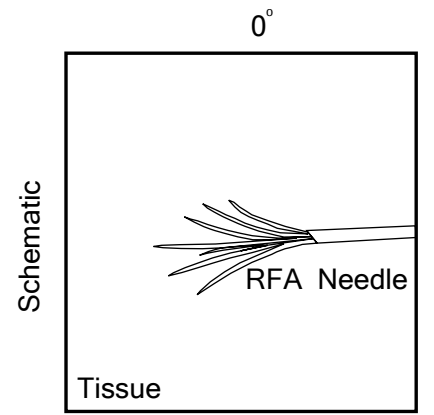

(a)

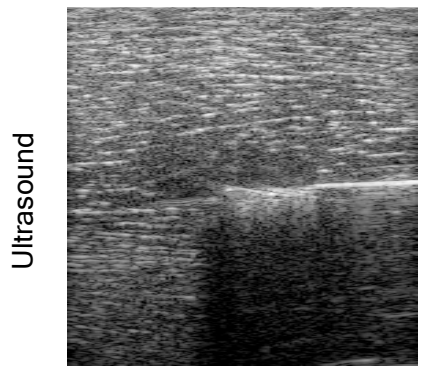

(d)

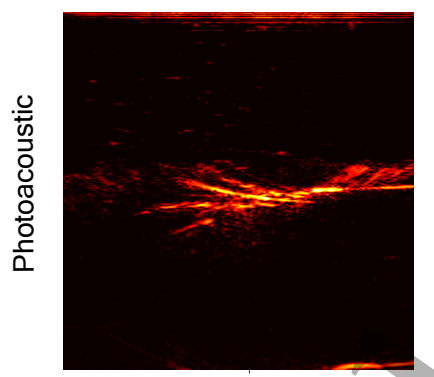

(g)

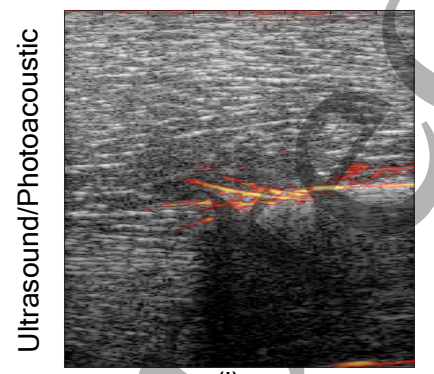

(j)

Photoacoustic [dB]

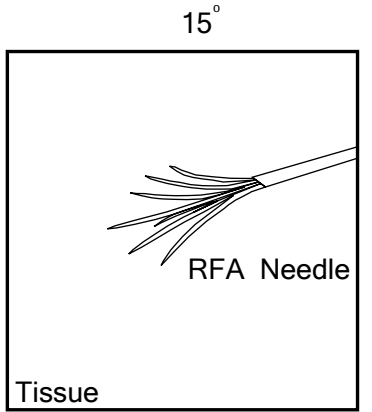

(b)

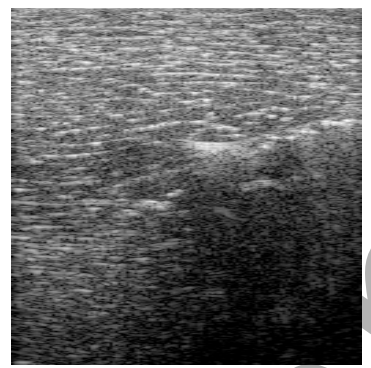

(e)

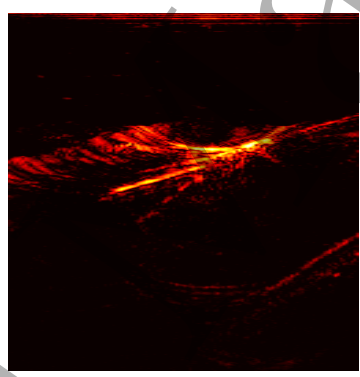

(h)

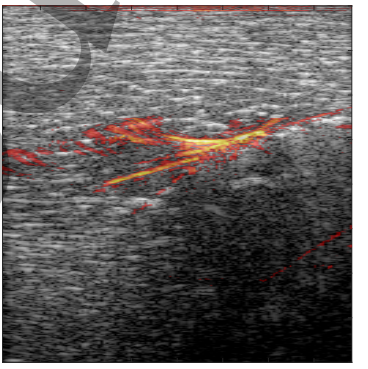

(k)

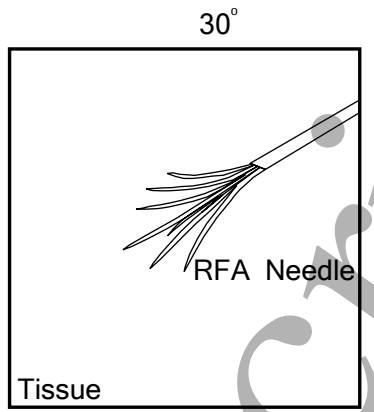

(c)

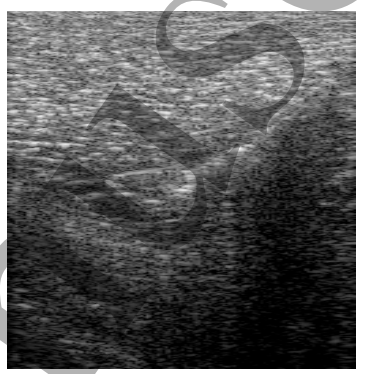

(f)

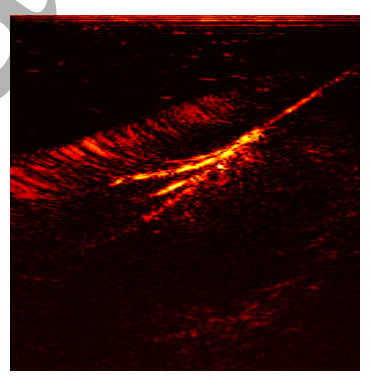

(i)

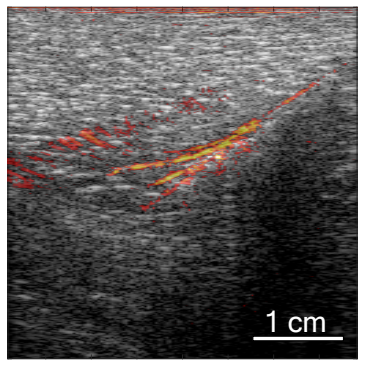

(I)

Figure 2. The radiofrequency ablation (RFA) needle inserted at different angles in chicken breast tissue. The schematic (a-c), ultrasound (d-f), photoacoustic (g-i) and combined ultrasound/photoacoustic (j-l) images of the RFA needle inserted at $0^{0}, 15^{0}$ and $30^{\circ}$ respectively. 
is slightly lower ranging from 3.2 to 3.7. Although RFA device visibility is poor in US images, it is rich in structural information of the tissue. This enhanced contrast of the RFA device from PA imaging can be overlaid on the structural image generated by US as shown in Fig. 2 (j-l). There are artifacts in both US and PA images. In the US image, the dominant artifact arises from strong acoustic shadowing from the metal needle seen below the needle in Fig. 2 (d-f). In PA images there are two main artifacts. The first appears below the actual RFA needle, mimicking its structure but at a steeper angle. This is due to a photoacoustically generated plane wave from the transducer surface due to light scattering onto the surface. The second artifact, which appears above the RFA needle is an artifact of the reconstruction. We discuss these artifacts in more detail in Section 4.

\begin{tabular}{|c|c|c|c|c|}
\hline \multirow{2}{*}{ Angle } & \multicolumn{2}{|c|}{$\begin{array}{c}\text { Photoacoustic } \\
\text { CNR (standard deviation) }\end{array}$} & \multicolumn{2}{c|}{ UNR (standard deviation) } \\
\cline { 2 - 5 } & Trocar & Tines & Trocar & Tines \\
\hline $0^{0}$ & $5.1 \pm 0.20$ & $8.9 \pm 0.06$ & $1.9 \pm 0.03$ & $1.4 \pm 0.04$ \\
\hline $15^{0}$ & $4.5 \pm 0.10$ & $7.5 \pm 0.13$ & $1.3 \pm 0.01$ & $1.6 \pm 0.02$ \\
\hline $30^{0}$ & $3.8 \pm 0.13$ & $6.0 \pm 0.20$ & $0.1 \pm 0.04$ & $0.7 \pm 0.06$ \\
\hline
\end{tabular}

Table 1. Contrast to Noise Ratio (CNR) of Photoacoustic (PA) and Ultrasound (US) images of RFA device trocar and tines for different angle of insertion. The CNR $\left(\left|\mu_{R O I}-\mu_{B c k}\right| / \sqrt{\sigma_{R O I}^{2}+\sigma_{B c k}^{2}}\right.$, where $\mu$ is mean and $\sigma$ is the standard deviation of pixels) calculation is performed over two image patches of $6 \times 6 \mathrm{~mm}$, one from the region of interest (ROI) and another from an adjacent background patch (Bck). Mean CNR and standard deviation are computed for three different locations along the trocar and tines.

\subsection{Guiding RFA Needle to Target Tissue}

The second experiment was conducted to study the capability of PA assisted needle guidance to a target region within the tissue. The target tissue was developed to have different acoustic and optical properties compared to normal tissue. Figure 3 (a) shows the target tissue containing India ink placed on a layer of chicken breast tissue. Two more layers of chicken tissue were stacked to form the imaging volume. The target tissue can be seen as a hypoechoic region in the US image in Fig. 3 (b) along with the three layers of chicken breast tissue. Due to optical absorption by the ink, the PA signal from the target tissue is high in Fig. 3 (c). PA frames showing RFA needle guidance to the target tissue and the deployment of tines is presented in $3(\mathrm{~d})$. The PA assisted RFA needle and tine deployment can be seen in video 1 in the supplementary material. After placing the needle in the target tissue, US and PA images were acquired. The US image of the target tissue with the RFA needle inside it is shown in Fig. 3 (e). The visibility of the needle and the tines are low under US imaging. Figure 3 (f) shows the PA image of the RFA needle and the tines deployed inside the target tissue. PA intensity of the target tissue boundary and the RFA needle is significantly higher than the surrounding 


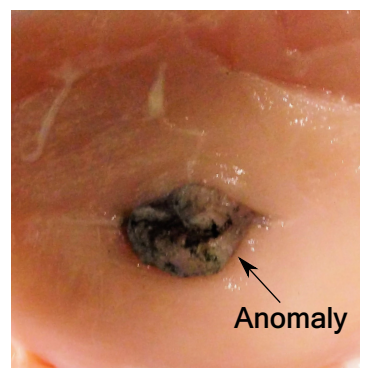

(a)

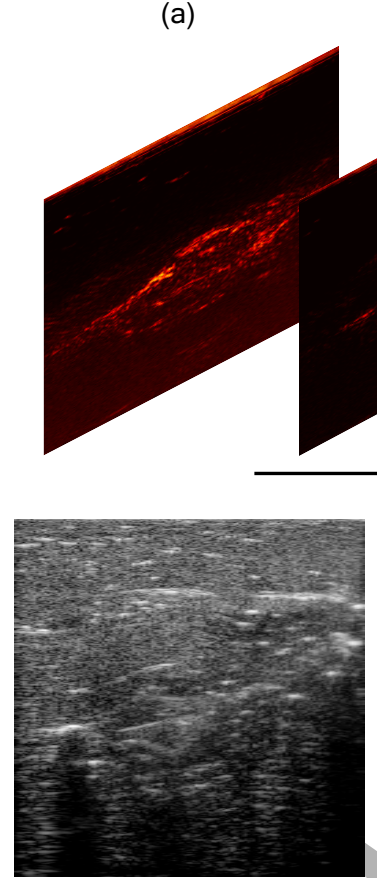

(e) -30 Photoacoustic [dB]

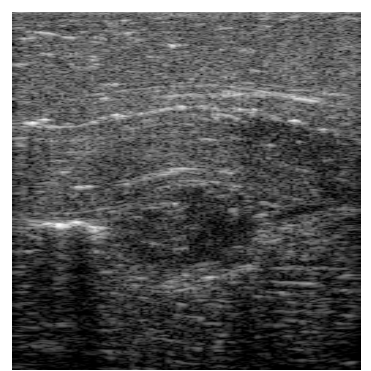

(b)
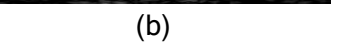

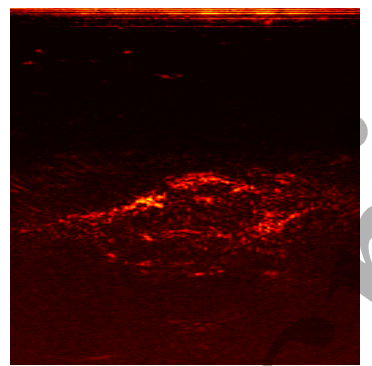

(c)
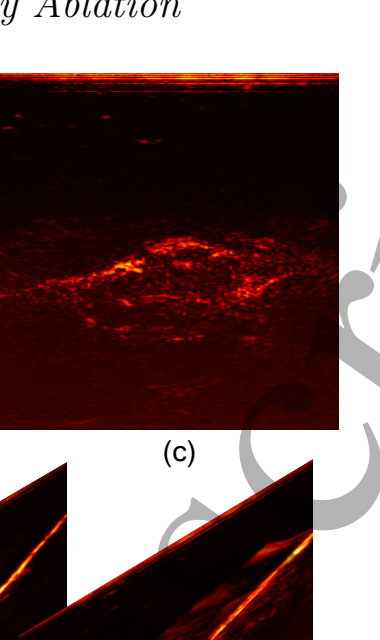

(d)

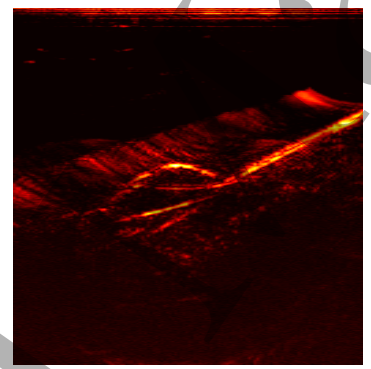

(f)

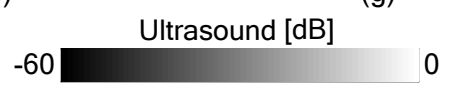

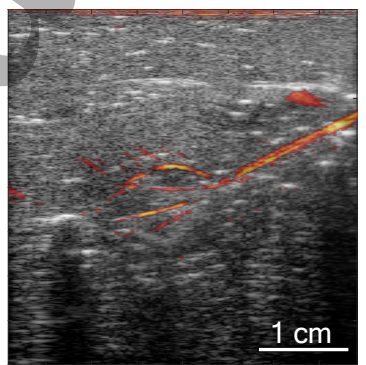

(g)

Figure 3. Guiding the radiofrequency ablation (RFA) needle to an anomalous target. (a) Chicken tissue with an anomalous target at center. (b) Ultrasound image showing hypoechoic anomalous region. (c) Photoacoustic image showing high intensity due to the absorbing anomaly. RFA needle inserted and deployed into the target, (d) imaged using ultrasound, (e) photoacoustic image and (f) combined PA-US image.

tissue. The US and PA artifact observed in the previous experiment in Fig. 2 can be also found in Fig. 3 (e) and (f) respectively. Figure 3 (g) shows the combined PA and US image with the PA enhanced image of the RFA needle and the target absorber in the tissue. Video 2 in the supplementary material shows combined real-time PA and US movie showing the RFA needle deployment into the target tissue.

\subsection{Mapping Blood Vessels}

In the third experiment, the mapping of blood vessels during RFA needle guidance was studied. Figure 4 (a) shows the schematic of the imaging volume with a tube filled with 
Photoacoustic Imaging in Percutaneous Radiofrequency Ablation

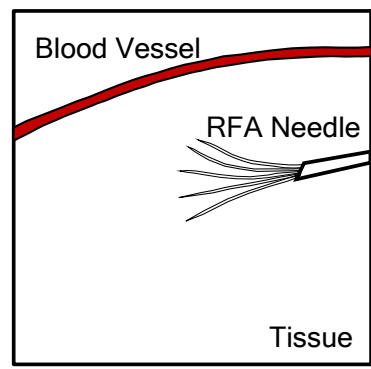

(a)

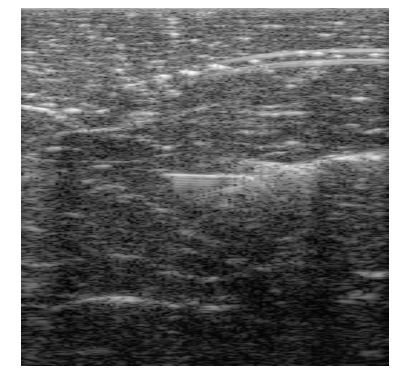

(b)

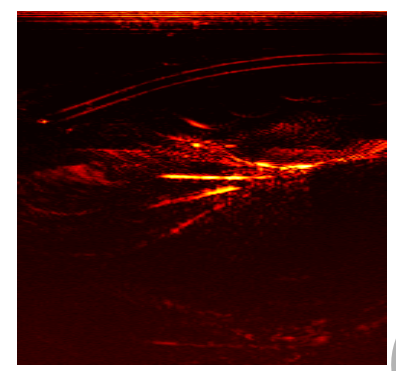

(c)

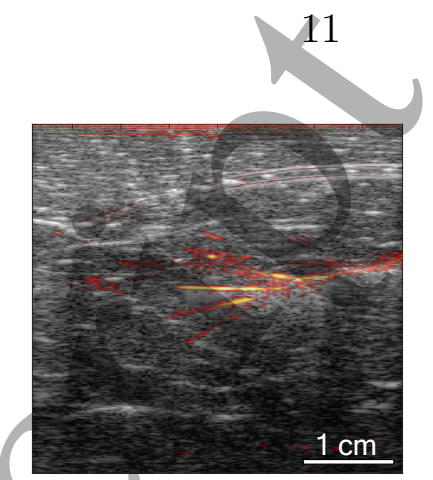

(d)

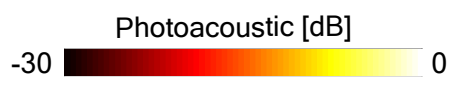

$-60$

Figure 4. Guiding the radiofrequency ablation (RFA) needle near large blood vessels. (a) Schematic shows the location of blood vessel mimicking phantom in chicken tissue and RFA needle. (b) Ultrasound image (c) Photoacoustic image and (d) Combined ultrasound and photoacoustic image.

human blood embedded in chicken breast tissue to mimic blood vessel. The figure also shows the location of the RFA needle inserted into the imaging volume. The US image in Fig. 4 (b) shows the inner and outer wall of the blood vessel. RFA needle and one of the tines is visible in the US image. Figure 4 (c) shows high PA intensity along the inner wall of the blood vessels. The oxygen saturation of the blood was changed from $47 \%$ to $88 \%$ which resulted in a drop in PA-intensity by $23 \%$ at $760 \mathrm{~nm}$. The RFA needle with four tines is clearly visible with high contrast in the PA image. A CNR value of $3.7 \pm 0.4$ was observed for blood vessels while that RFA needle was $16.3 \pm 3.5$. Combined image showing blood vessel, RFA needle and tissue structure in figure 4 (d). Video 3 in supplementary material shows the real-time PA frames showing change in intensity with oxygen saturation of the blood.

\subsection{Percutaneous Needle Guidance to Liver Tissue}

In the fourth experiment, percutaneous RFA needle guidance through soft tissue to the liver was investigated. Figure 5 (a) shows the schematic with chicken breast tissue to mimic soft tissue on the top and bovine liver below. The location of the RFA needle is also shown in the figure. Figure 5 (b) shows the US image with a hyperechoic soft tissue region compared to the liver. The soft tissue liver boundary is clearly visible in the US image. However, the RFA needle is not clear in the image. Fig. 5 (c) shows PA image overlaid on US image. High PA intensity from the RFA needle co-registered on the US image combines structural information from the US showing tissue boundary and accurate location of the needle. A maximum imaging depth of $16.7 \mathrm{~mm}$ was observed with respect to the transducer, which consists of $9.4 \mathrm{~mm}$ through the soft tissue and $7.3 \mathrm{~mm}$ through the liver. The CNR of PA image for the RFA trocar and tines are respectively $3.2 \pm 0.21$ (standard deviation) and $7.2 \pm 0.33$. While CNR for US image of the trocar is $0.3 \pm 0.04$ and $1.0 \pm 0.06$ for the tines. Video 4 in the supplementary 
Photoacoustic Imaging in Percutaneous Radiofrequency Ablation

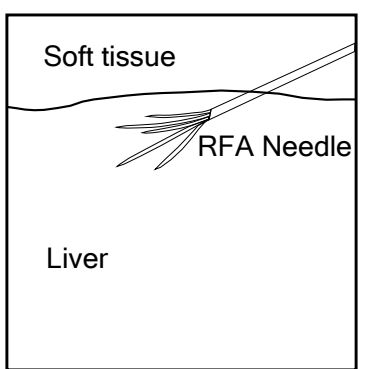

(a)

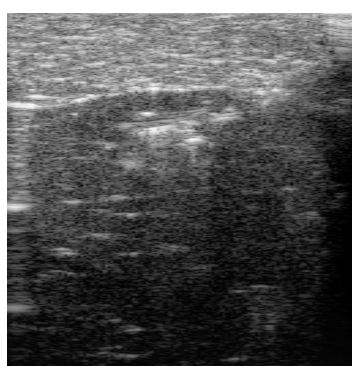

(b)

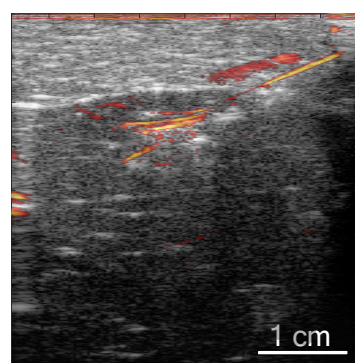

(c)

-30 Photoacoustic [dB] 0

$-60$

Figure 5. Combined photoacoustic-ultrasound assisted needle guidance to the liver. (a) Schematic showing liver, soft tissue and needle location (b) Showing the ultrasound image of the RFA needle inserted to the bovine liver through a layer of chicken tissue.

(c) Combined PA-US image shows improved visibility of needle and tines.

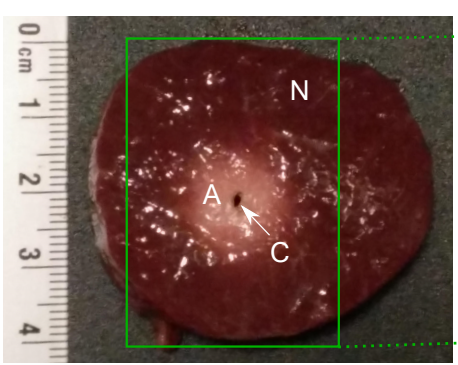

(a)

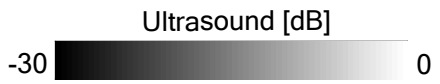

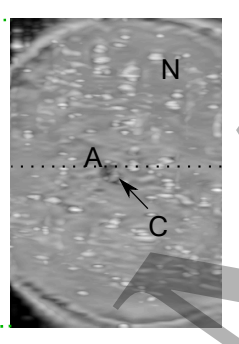

(b)

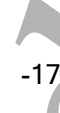

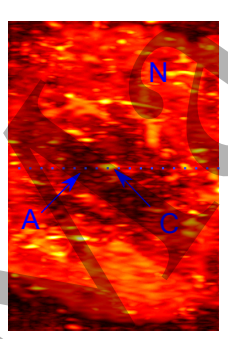

(c)

Photoacoustic [dB]

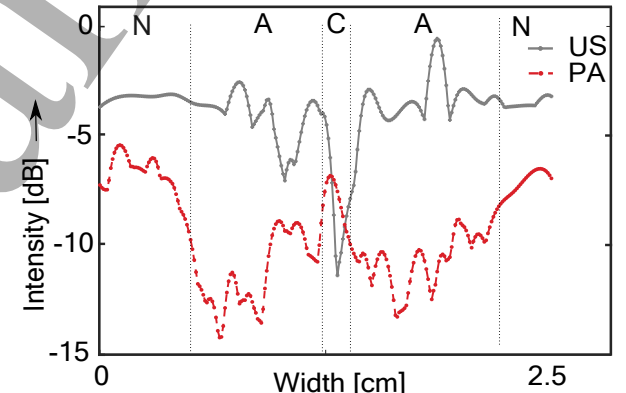

(d)

A - Ablated tissue

C - Charring

Figure 6. Imaging ablated bovine liver (a) Photograph of the liver slice with ablation lesion at the center. The green box marks the imaging area. (b) Maximum Intensity Projection (MIP) of yolumetric ultrasound image along the depth direction. (c) MIP of photoacoustic image. (d) Photoacoustic and ultrasound line profile along the center of the ablated zone (marked). The native, ablated and charred regions are also marked in the line profile.

material shows the tine deployment into the liver tissue. The PA reconstruction artifact can also be seen in the images.

\subsection{Ablation Imaging}

The final experiment was conducted to study the nature of the PA signal from ablated and native tissue. Figure 6 (a) shows the photograph of the ex vivo bovine liver tissue slice with the ablated center. The green rectangle marks the imaged region. The hole at the center of the ablated region was due to the RFA needle insertion, and there is a visible charring observed around the needle. The US Maximum Intensity Projection (MIP) image in Fig. 6 (b) shows the boundary of the tissue as well as the hole at the 


\section{Photoacoustic Imaging in Percutaneous Radiofrequency Ablation}

center of the sample. However, there is no significant change in US intensity observed between ablated and native tissue. The MIP PA image in Fig. 6 (c) shows a reduction in intensity from the ablated tissue compared to the native tissue. The charring is also visible as a high PA signal at the center of the ablated region. A difference of $5.2 \pm 0.5$ $\mathrm{dB}$ (standard deviation) in the PA intensity was observed between native and ablated tissue. While ablated tissue and charring differ by $1.9 \pm 0.6 \mathrm{~dB}$. The change in intensity between native, ablated and charred tissue is also evident in the PA intensity profile through the center of the sample in Fig. $6(\mathrm{~d})$. The drop in US intensity in the line profile in Fig. 6 (d) is from the hole at the center of the sample. The 3D US and PA images (video $5 \& 6$ ) of the tissue are included in the supplementary material.

\section{Discussion}

Key challenges in percutaneous RFA treatment are accurate targeting of the needle and determination of the amount of ablated tissue under US image guidance [30]. Accurate feedback from the imaging modality can improve the RFA treatment outcome [8]. We investigated the proof-of-concept of PA imaging in combination with US imaging, for the guidance of RFA procedures in the laboratory, in experiments simulating the clinical setting. Scenarios such as mapping blood vessels, guilding RFA needle to a target tissue, and identifying the boundary of the ablated tissue, which surgeons perform during an RFA procedure were studied under PA and US image assistance on ex vivo samples. Our preliminary results show that the PA imaging is capable of (i) enhancing the RFA needle visibility, (ii) mapping blood vessels and (iii) differentiating the ablated tissue.

We have exploited the optical absorption of the RFA needle and blood vessels, and differences in optical absorption between normal, anomalous and ablated tissue to demonstrate the capability of PA image-guided RFA procedures. The key finding is that the RFA device and its tines (with high optical absorption imparted using paint) can be visualized accurately using PA imaging. This vastly improved visibility with the PA imaging can be useful in needle guidance especially when the needle is inserted at an angle with respect to the transducer. At steeper angles, the RFA needle was hard to visualize in US imaging (Fig. $2(\mathrm{~d}-\mathrm{f})$ ). The reason for this is that the US undergoes specular reflection from the needle at a steep angle, and propagates away from the linear aperture and goes undetected [31]. However, PA images (Fig. 2 (gi)) showed considerably improved visibility with more than two-fold improvement in contrast compared to US images for all insertion angles for the needle trocar. In PA imaging, acoustic waves generated from the needle surface propagate at the same angle as that of the needle and get detected by the transducer. This improvement in contrast is also evident for the RFA tines (Fig. 2 (g-i)) with more than four times the contrast in PA images compared to US. It can be also noted that there is no significant drop in PA intensity from the needle with a change in angle. However, the small change in CNR value in Table. 1 for PA images is due to the presence of artifact in the images, which are dominant at larger angles. This improved visibility of the RFA needle in the PA 


\section{Photoacoustic Imaging in Percutaneous Radiofrequency Ablation}

imaging compared to the US can help surgeons to guide the needle accurately and at steeper angles. The capability of needle guidance under PA assistance has earlier been demonstrated with biopsy needles $[32,33]$ which we have extending to RFA needles in this work. The results from the experiment exploring the capability of needle guidance to a target as demonstrated in Fig. 3, also emphasize the advantage of PA for needle guidance. The results (Fig. 3) demonstrate the possibility of guiding the RFA needle to tumor tissue and accurate deployment of tines. Although in this proof-of-concept study we used an artificial absorbing target, it is known that an optical absorption contrast exists between tumor and normal tissue [19] which can be exploited for locating the target for guidance. Intrinsic contrast from increased vascularity associated with cancer growth is widely used in PA imaging to identify tumor [34]. Results from section 3.3, provide indication of expected contrast from blood at different oxygen concentration. With proper selection of wavelength, the target tumor tissue can be highlighted which was demonstrated in a similar PA setting [35].

A real-time map of neighboring vessels during the needle guidance is of paramount importance in RFA to avoid damage to the blood vessels [36]. Further, ablation near larger blood vessels suffers heat losses to the cooler circulating blood (heat sink effect) resulting in incomplete treatment [36]. Hence it is essential to delineate blood vessels and provide information for optimizing thermal dosimetry to compensate the heat sink effect. Pre-ablation MR or Doppler US images are used to map blood vessels and to define the needle path in RFA procedures. We have demonstrated (in Fig. 4) that real-time PA imaging of blood vessel in tissue during RFA needle guidance is possible. The contrast of RFA needle in Fig. 4 is three time higher compared to blood. This might pose difficulty when the blood vessels are placed next to the needle. A possible alternative is to modulate the absorption of the RFA needle with a spectral signature for multi-wavelength PA imaging. High PA signals from the blood carrying tubes are observed from the inner, wall and not from the whole tube in Fig. 4. This is due to the band-limited nature of the transducer filtering out low-frequency signals [37].

Although RFA is established a clinical practice in liver cancer management, high local recurrence rates have been reported [8]. There is a need to improve the accuracy of the procedure. We have demonstrated percutaneous RFA needle guidance to the liver (Fig. 5) and ablation visualization (Fig. 6) using PA imaging. The potential of combined US and PA imaging in the percutaneous RFA needle guidance to the liver through soft tissue is evident in Fig. 5. US images can be used to provide structural images such as the boundary of liver and soft tissue in this case, while the co-registered photoacoustic image can enhance the visibility of highly absorbing structures like the RFA needle (Fig. 5 (c)). The advantage of PA imaging is that it can work as an add-on to conventional US imaging systems as in Fig. 1 and hence it might be easy to translate to the clinical setting [38]. The penetration depth of $7.3 \mathrm{~mm}$ in the liver is a limitation of the current illumination method from outside the tissue, considering the laser safety limits. Given this limitation, the demonstrated approach can be used for superficial liver tumors. Further, to enable deep tissue PA imaging in highly absorbing organs like 
liver, we are developing an interstitial illumination where light can be delivered at the region of interest [39].

The final step in the RFA procedure is to determine the extent of the thermal lesion, to judge ablation success and make a decision on whether to further apply the procedure. Conventionally US imaging is often used to estimate the ablation boundary based on high scattering from bubbles formed in the necrosis zone. However, imaging bubbles to estimate thermal lesion size is an indirect method and can result in an error due to shadowing. The optical property change with heating of tissue is utilized to differentiated ablated and normal tissue in Fig. 6 (c). An observed PA intensity difference of $5 \mathrm{~dB}$ indicates the potential of using this technology for ablation monitoring. Compared to native tissue, a reduction in intensity was observed. This observation agrees with previous ex vivo studies which reported a reduction in optical absorption and an increase in the scattering of ablated tissue compared to native in the NIR wavelengths [40, 23]. However, in in vivo this might vary due to increased vascularity around the ablated region, which can potentially give a high PA signal. Given the reduction in PA intensity with ablation, there are challenges in simultaneous visualization of the RFA device and the ablation zone. The high intensity from the device with the artifacts can overwhelm the intensity difference between the ablated and native tissue. Currently, we are working with an imaging protocol where ablation imaging is performed after withdrawing the RFA device. Another possibility is to minimize the signal from the needle with the use of an appropriate coating or modulate the RFA device with another coating which has a wavelength specific absorption. Further, Iskander et al. [21] proposed the use of dual wavelength imaging to utilize the differential contrast of ablated and native tissue. These aspects of using multiple wavelength images to form an ablation map need to be explored for liver ablation. We are also working on multi-wavelength PA imaging approach to provide a positive contrast to the ablated tissue.

This study while demonstrating the feasibility of using PA imaging together with US imaging in the setting of RFA is a preliminary one and further investigations are required. One of the problems that we faced was the occurrence of artifacts. Figure 2 $(\mathrm{g}-\mathrm{i})$ shows two types of PA artifacts. The first is a weak artifact below the needle with the same structure as the needle, but appearing at a steeper angle. This artifact is a reflection from the needle, of an US plane wave emitted from the face of the US transducer by light falling on it. The transducer front surface comprises a dark matching layer and absorbs light backscattered from the sample during PA illumination setting up a laser-induced US plane wave. This propagates into the sample and reflects off the needle to be detected. A study we conducted to understand the cause of the artifact is provided in the supplementary material. A non-absorbing matching layer can eliminate this artifact. The second artifact is the one above the needle (Fig. 2). Our analysis provided in the supplementary material shows that this artifact is most likely a backprojection artifact. When the PA intensity is high at certain positions, this is smeared out along the circular backprojection paths leading to a strong signal elsewhere 


\section{Photoacoustic Imaging in Percutaneous Radiofrequency Ablation}

in the image. Other groups have also observed this PA artifact in their results [32] [41], [42], but have not explicitly studied their origin or eliminated it. More studies are required to understand the artifact better and develop post-processing steps to remove it.

It can be observed that the tines at the lower portion of the needle in Fig. 2 (g-i) are less visible in PA images with multiple structures above forming obstacles for the sound path. This is a limitation with the use of linear array with its limited recording aperture where we cannot detect PA signal propagating from all the angles. The US images in Fig. $2(\mathrm{~d}-\mathrm{f})$ shows a shadow artifact. This can be rectified to a large extent by using spatial compounding of B-mode images obtained by steering the beam at different angles. However, in this study, we prefer to use a B-mode image without spatial compounding for the ease of combined PA and US imaging [43]. In all the experiments the RFA tines (not the trocar) were coated to improve absorption. The difference in the PA signal from the coated and bare part of the needle can be observed in Fig. 2 (g-i) and the CNR values in Table 1. Seyeral materials to modulate PA signals from metals have been previously investigated [44]. However, a specific study needs to be conducted in the context of RFA based on electrical conductivity, optical property as well as biocompatibility of the coating material. Due to the high absorption of liver tissue, illumination from the skin surface may not sufficient to permit PA imaging guidance of RFA in this organ. However, there is a large scope in applying the same strategy as in this work in laparoscopic RFA, with the use of optical fibers and laparoscopic US imaging. Further, interstitial illumination methods which bring the light close to the target organ and tissues can address this aspect. Finally, the optical and acoustic properties of the liver during and after ablation need to be further studied to develop a PA imaging system for deep tissue ablation monitoring.

\section{Conclusion}

The results demonstrate the feasibility of using photoacoustic (PA) imaging together with US imaging in the context of radiofrequency ablation (RFA). We have shown that an imaging platform based on PA and US imaging can be used to visualize the RFA device, and locate the target/anomalous tissue to enable improved guidance. Further the approach can be used to image blood vessels in the vicinity of the needle during insertion; this also permits developing awareness of possible heat-sink effects in developing a thermal lesion at the target tissue. Finally, the potential of PA imaging in differentiating ablated and native tissue due to the change in optical properties are shown in the results. Future work will concentrate on interstitial illumination for deep tissue imaging and multi-wavelength imaging for ablation monitoring. 


\section{Acknowledgments}

This work is funded by a joint grant from the Netherlands Organisation for Scientific Research (NWO)/the Netherlands Organisation for Health Research and Development (ZonMw) and the Department of Biotechnology (Government of India) under the program Medical Devices for Affordable Health (MDAH) as Project Imaging Needles (Grant Number 116310008). Authors acknowledge the Faculty of Science and Technology (TNW) and the Netherlands Organisation for Scientific Research (NWO) for further support via the SEO (Stimulering Europees Onderzoek) funds. Authors acknowledge contributions of Vinay Parameshwarappa in the initial studies, and the MIRA Strategic Call 2016 for a seed funding that could start this work.

\section{References}

[1] J. Chang and D. W. Rattner, "History of minimally invasive surgical oncology," Surgical Oncology Clinics (2018).

[2] J. L. Hinshaw, M. G. Lubner, T. J. Ziemlewicz, et al., "Percutaneous tumor ablation tools: microwave, radiofrequency, or cryoablationwhat should you use and why?," Radiographics 34(5), 1344-1362 (2014).

[3] R. Lencioni and L. Crocetti, "Local-regional treatment of hepatocellular carcinoma," Radiology 262(1), 43-58 (2012).

[4] G. D. Dodd, M. C. Soulen, R. A. Kane, et al., "Minimally invasive treatment of malignant hepatic tumors: at the threshold of a major breakthrough," Radiographics 20(1), 9-27 (2000).

[5] L. M. Sutherland, J. A. Williams, R. T. Padbury, et al., "Radiofrequency ablation of liver tumors: a systematic review," Archives of Surgery 141(2), 181-190 (2006).

[6] Y. Ni, S. Mulier, Y. Miao, et al., "A review of the general aspects of radiofrequency ablation," Abdominal imaging 30(4), 381-400 (2005).

[7] S. Mulier, Y. Ni, J. Jamart, et al., "Local recurrence after hepatic radiofrequency coagulation: multivariate meta-analysis and review of contributing factors," Annals of surgery 242(2), 158 (2005).

[8] B. M. Künzli, P. Abitabile, and C. A. Maurer, "Radiofrequency ablation of liver tumors: Actual limitations and potential solutions in the future," World journal of hepatology 3(1), 8 (2011).

[9] W. Xia, "Emerging clinical applications of photoacoustic imaging," Journal of Biomedical Imaging and Bioengineering 1(1) (2017).

[10] Y. Minami and/M. Kudo, "Imaging modalities for assessment of treatment response to nonsurgical hepatocellular carcinoma therapy: contrast-enhanced US, CT, and MRI," Liver Cancer 4(2), 106-114 (2015).

[11] L.-H. Lee, J.-I. Hwang, Y.-C. Cheng, et al., "Comparable outcomes of ultrasound versus computed tomography in the guidance of radiofrequency ablation for hepatocellular carcinoma," PloS one 12(1), e0169655 (2017).

[12] C. H. Cha, F. T. Lee Jr, J. M. Gurney, et al., "CT versus sonography for monitoring radiofrequency ablation in a porcine liver," American Journal of Roentgenology 175(3), 705-711 (2000).

[13] D. Cioni, R. Lencioni, S. Rossi, et al., "Radiofrequency thermal ablation of hepatocellular carcinoma: using contrast-enhanced harmonic power doppler sonography to assess treatment outcome," American Journal of Roentgenology 177(4), 783-788 (2001).

[14] T. Hori, K. Nagata, S. Hasuike, et al., "Risk factors for the local recurrence of hepatocellular carcinoma after a single session of percutaneous radiofrequency ablation," Journal of gastroenterology 38(10), 977-981 (2003). 
Photoacoustic Imaging in Percutaneous Radiofrequency Ablation

[15] M. Xu and L. V. Wang, "Photoacoustic imaging in biomedicine," Review of scientific instruments $\mathbf{7 7}(4), 041101$ (2006).

[16] K. J. Francis, B. Chinni, S. S. Channappayya, et al., "Characterization of lens based photoacoustic imaging system," Photoacoustics 8, 37-47 (2017).

[17] J. L. Su, R. R. Bouchard, A. B. Karpiouk, et al., "Photoacoustic imaging of prostate brachytherapy seeds," Biomedical optics express 2(8), 2243-2254 (2011).

[18] M. Toi, Y. Asao, Y. Matsumoto, et al., "Visualization of tumor-related blood vessels in human breast by photoacoustic imaging system with a hemispherical detector array," Scientific reports 7, 41970 (2017).

[19] S. L. Jacques, "Optical properties of biological tissues: a review," Physics in Medicine 83 Biology 58(11), R37 (2013).

[20] N. Dana, L. Di Biase, A. Natale, et al., "In vitro photoacoustic visualization of myocardial ablation lesions," Heart Rhythm 11(1), 150-157 (2014).

[21] S. Iskander-Rizk, P. Kruizinga, A. F. van der Steen, et al., "Spectroscopic/photoacoustic imaging of radiofrequency ablation in the left atrium," Biomedical optics express 9(3), 1309-1322 (2018).

[22] J. Rebling, F. J. O. Landa, X. L. Deán-Ben, et al., "Integrated catheter for simultaneous radio frequency ablation and optoacoustic monitoring of lesion progression," Optics letters 43(8), 1886-1889 (2018).

[23] P. Lanka, K. Francis, H. Kruit, et al., "Monitoring radiofrequency ablation of biological tissue using broadband time-resolved diffuse optical spectroscopy," in Diffuse Optical Spectroscopy and Imaging VII, 11074, 110742M, International Society for Optics and Photonics (2019).

[24] M. K. A. Singh, W. Steenbergen, and S. Manohar, "Handheld probe-based dual mode ultrasound/photoacoustics for biomedical imaging," in Frontiers in Biophotonics for Translational Medicine, 209-247, Springer (2016).

[25] M. K. A. Singh, V. Parameshwarappa, E. Hendriksen, et al., "Photoacoustic-guided focused ultrasound for accurate visualization of brachytherapy seeds with the photoacoustic needle," Journal of biomedical optics 21(12), 120501 (2016).

[26] K. J. Francis and S. Manohar, "Photoacoustic assisted device guidance and thermal lesion imaging for radiofrequency ablation," in ECBO 2019: Opto-Acoustic Methods and Applications in Biophotonics, 11077, 40, International Society for Optics and Photonics (2019).

[27] J. Kim, S. Park, Y. Jung, et al., "Programmable real-time clinical photoacoustic and ultrasound imaging system," Scientific reports 6, 35137 (2016).

[28] T. Gould, Q. Wang, and T. J. Pfefer, "Optical-thermal light-tissue interactions during photoacoustic breast imaging," Biomedical optics express 5(3), 832-847 (2014).

[29] Angiodynamics, "Rita starburst - instructions for use," https://www.angiodynamics.com/img/ resources/StarBurst_Xli-e_IFU-366770.pdf (2010). Last accessed 15 Feburay 2019.

[30] H. Takahashi, M. Akyuz, E. Aksoy, et al., "Local recurrence after laparoscopic radiofrequency ablation of malignant liver tumors: Results of a contemporary series," Journal of surgical oncology 115(7), 830-834 (2017).

[31] G. Reusz, P. Sarkany, J. Gal, et al., "Needle-related ultrasound artifacts and their importance in anaesthetic practice," British journal of anaesthesia 112(5), 794-802 (2014).

[32] W. Xia, M. K. A. Singh, E. Maneas, et al., "Handheld real-time led-based photoacoustic and ultrasound imaging system for accurate visualization of clinical metal needles and superficial vasculature to guide minimally invasive procedures," Sensors (Basel, Switzerland) 18(5) (2018).

[33] D. Piras, C. Grijsen, P. Schutte, et al., "Photoacoustic needle: minimally invasive guidance to biopsy," Journal of biomedical optics 18(7), 070502 (2013).

[34] D. Hanahan and R. A. Weinberg, "Hallmarks of cancer: the next generation," cell 144(5), 646-674 (2011).

[35] R. Li, P. Wang, L. Lan, et al., "Assessing breast tumor margin by multispectral photoacoustic tomography," Biomedical optics express 6(4), 1273-1281 (2015).

[36] L. Thanos, S. Mylona, P. Galani, et al., "Overcoming the heat-sink phenomenon: successful 
1

2

3

4

5

6

7

8

9

Photoacoustic Imaging in Percutaneous Radiofrequency Ablation

radiofrequency thermal ablation of liver tumors in contact with blood vessels," Diagnostic and Interventional Radiology 14(1), 51 (2008).

[37] R. G. Kolkman, N. Bosschaart, B. Kok, et al., "Photoacoustic imaging of valves in superficial veins," Lasers in Surgery and Medicine: The Official Journal of the American Society for Laser Medicine and Surgery 38(8), 740-744 (2006).

[38] M. W. Schellenberg and H. K. Hunt, "Hand-held optoacoustic imaging: A review," Photoacoustics (2018).

[39] K. J. Francis, E. Rascevska, and S. Manohar, "Annular illumination photoacoustic probe for needle guidance in medical interventions," in ECBO 2019: Opto-Acoustic Methods and Applications in Biophotonics, 11077, 20, International Society for Optics and Photonics (2019).

[40] C.-T. Germer, A. Roggan, J. P. Ritz, et al., "Optical properties of native and coagulated human liver tissue and liver metastases in the near infrared range," Lasers in Surgery and Medicine: The Official Journal of the American Society for Laser Medicine and Surgery 23(4), 194-203 (1998).

[41] K. Sivasubramanian, V. Periyasamy, and M. Pramanik, "Non-invasive sentinel lymph node mapping and needle guidance using clinical handheld photoacoustic imaging system in small animal," Journal of biophotonics 11(1), e201700061 (2018).

[42] M. A. L. Bell and J. Shubert, "Photoacoustic-based visual servoing of a needle tip," Scientific reports $\mathbf{8}(1), 15519$ (2018).

[43] K. J. Francis, B. Chinni, S. S. Channappayya, et al., "Multiview spatial compounding using lensbased photoacoustic imaging system," Photoacoustics 13, 85-94 (2019).

[44] T. M. Mitcham, K. A. Homan, W. Frey, et al., "Modulation of photoacoustic signal generation from metallic surfaces," Journal of biomedical optics 18(5), 056008 (2013).

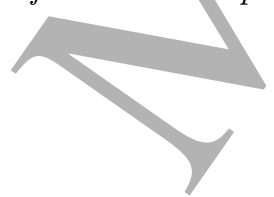

8

